\title{
Early Japanese contributions to space weather research (1945-1960)
}

\author{
A. Nishida \\ Retired from: Institute of Space and Astronautical Science, Japan Aerospace Exploration Agency, Japan \\ Home: 2-9-25 Tamagawa-Gakuen, Machida, Tokyo 194-0041, Japan \\ This paper was published in "Climate and Weather of the Sun-Earth System (CAWSES): Selected Papers from the 2007 \\ Kyoto Symposium”, edited by: Tsuda, T., Fujii, R., Shibata, K., and Geller, M. A., TERRAPUB, Tokyo, 2009 and reprinted \\ in HGSS by the kind permission of the original publisher.
}

Received: 1 March 2010 - Accepted: 19 March 2010 - Published: 1 April 2010

\begin{abstract}
Major contributions by Japanese scientists in the period of 1945 to 1960 are reviewed. This was the period when the foundation of the space weather research was laid by ground-based observations and theoretical research. Important contributions were made on such subjects as equatorial ionosphere in quiet times, tidal wind system in the ionosphere, formation of the F2 layer, VLF propagation above the ionosphere, and precursory phenomena (type IV radio outburst and polar cap absorption) to storms. At the IGY (1957, 1958), research efforts were intensified and new programs in space and Antarctica were initiated. Japanese scientists in this discipline held a tight network for communication and collaboration that has been kept to this dav.
\end{abstract}

\section{Introduction}

On the occasion of the International CAWSES Symposium held at Kyoto in October 2007, I presented a short history of research in this field that was made in Japan over the period of 15 years from 1945 to 1960 . As the designation of the research discipline, "Space Weather" followed "Solar Terrestrial Physics" which had developed from the classical disciplines of ionospheric physics, geomagnetism, solar physics and cosmic ray physics. The year 1945 was when the World War II ended and basic research was reactivated, and the year 1960 was roughly the time when in-situ observations by space vehicles became a common tool of research and the frontier of the research rapidly expanded.

In these 15 years the research depended on remote sensing observations from the ground. Structures and dynamics of the ionosphere and above were inferred from the analyses of such data and new observations were designed to advance the understanding. Most of the basic concepts of the solar terrestrial physics that developed out of these efforts stand today as the basis of Space Weather research. This paper reviews important contributions made by Japanese scientists to this development.

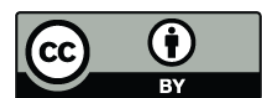

Correspondence to: A. Nishida (hirosoph@dj.mbn.or.jp)
Before highlighting individual contributions in the following chapters, we should note that the Japanese scientists in this discipline held a tight network of communication and collaboration. Already in 1946 the Special Committee on the Ionosphere was established in the Science Council of Japan. Receiving the grants from the government to support travels, scientists nationwide met together regularly to discuss the latest achievements and to plan for collaborative programs. "Report of Ionosphere Research in Japan" that was inaugurated in 1947 was the publication from this Committee. The Committee is still active (though the title has changed) and being run by the Solar Terrestrial Environment Laboratory of the Nagoya University. While the Ionosphere Committee was run by public support, the Society of Terrestrial Magnetism and Electricity was founded in 1947 as an organization of individual scientists in this field. Its publication, "Journal of Geomagnetism and Geoelectricity", was inaugurated in 1949. Society of Geomagnetism, Earth and Planetary Sciences (SGEPSS) is the current name of the same Society, and "Earth, Planets and Space" is the successor of JGG.

The author himself who enrolled in the graduate school in 1958 was a late comer to the generation of scientists who were active in the 1945-1960 period. But I remember how exciting it was to attend the meetings of the above Committee and Society and listen to fresh outcomes of the latest research efforts. For writing this article I went through the literature of the time and was impressed over again by the world-class contributions made by our predecessors. 


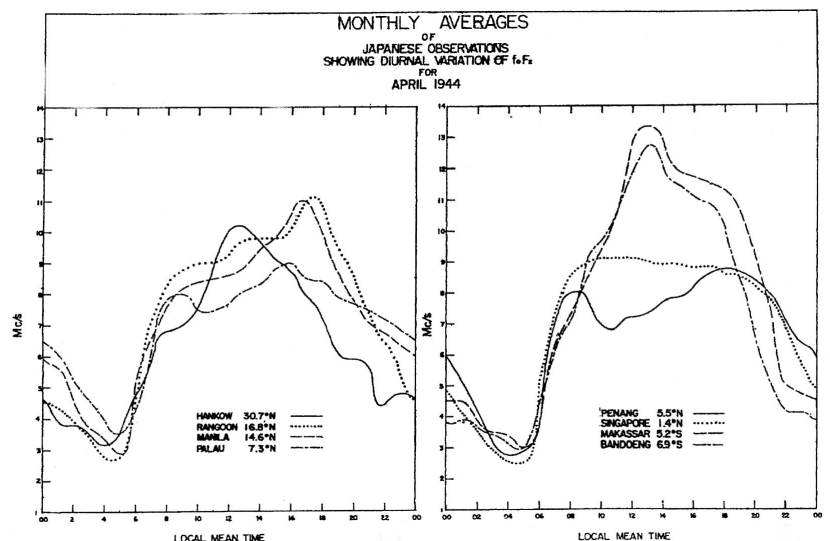

Figure 1. Daily variations of $f o \mathrm{~F} 2$ observed at eight stations at or near the geomagnetic equator in 1944. Noon time peak is absent or weak at stations around the geomagnetic equator. This diagram is taken from "Report of Japanese Research on Radio Wave Propagation," published by Pacific Office of the Chief Signal Officer, General Headquarters, United States Army Forces, May 1946.

\section{Prologue}

Ionospheric research had been conducted intensively from earlier times in Japan because it was necessary to monitor and forecast ionospheric conditions in these days of the short wave communication. The ionospheric warning was particularly important for Japan because the radio waves to/from North America and Europe traverse high latitudes and are susceptible to ionospheric disturbances. In addition to this, during the wartime when the Japanese forces were deployed to South-East Asia the conditions of the equatorial ionosphere had to be monitored in order to select the optimum frequency for radio communications. For the latter purpose, fourteen stations were built, spanning the equatorial region and low latitudes.

A major outcome from this low-latitude network of stations was the discovery of the equatorial anomaly in $f o \mathrm{~F} 2$ that is the measure of the peak electron density at the F2 layer. Figure 1 shows the $f o \mathrm{~F} 2$ versus local time at eight stations. While the diurnal variation of $f_{o} \mathrm{~F} 2$ peaked in the daytime at most of these stations, there were exceptions around the geomagnetic equator. At Singapore (geomagnetic latitude: $8.9^{\circ}$ ) and Penang $\left(4.7^{\circ}\right), f o \mathrm{~F} 2$ was flat in the daytime and were lower than the values that were observed further away from the geomagnetic equator. This feature was named later as Equatorial Anomaly by Appleton. ("Report of Japanese Research on Radio Wave Propagation", published from General Headquarters, United States Army Forces, Pacific Office of the Chief Signal Officer, May 1946, gives a fair account of this activity. D. K. Bailey was instrumental in compiling this report and helping the resumption of ionospheric research in the post-war Japan. See also a review article on the equatorial F-layer by Rishbeth (2000).)
In geomagnetic research, electric current systems in the ionosphere were studied by using geomagnetic data obtained during the second polar year. Daily variations in geomagnetically quiet days, $S q$, were studied extensively by Hasegawa who was attracted to this phenomenon while he was assigned to monitoring the magnetic disturbance accompanying the volcanic activity. He found that the focus of this current vortex was not fixed in latitude changing its position from day to day (Hasegawa, 1936). He was also aware that $S q$ had an anomalously large amplitude at the geomagnetic equator and noted this phenomenon (that is, the equatorial enhancement), as one of the important questions to solve. Concerning the geomagnetic disturbances, Hatakeyama made a pioneering study of the magnetic bays. He showed that global distribution of the bay disturbance was essentially the same as that of the diurnal part of magnetic storms, and interpreted the disturbance in terms of the intensification of the ionospheric current due to enhancement of the electric conductivity by the charged corpuscles from the sun. The electromotive force provided by the dynamo effect of the atmospheric motion was thought to be the same for both quiet and disturbed times (Hatakeyama, 1938).

\section{Electrodynamics of the ionosphere in quiet times}

The equatorial anomaly of $f o \mathrm{~F} 2$ and the equatorial enhancement of $S q$ were explained using the concepts of plasma drift and Hall conductivity.

\subsection{Equatorial anomaly of foF2}

The geomagnetic daily variation $S q$ had been considered to result from the current generated in the ionospheric $\mathrm{E}$ region by the dynamo action of the atmospheric tide. When the atmosphere is in motion with velocity $v$ relative to the Earth the dynamo electric field $v \times B$ is generated where $B$ is the magnetic field. The total electric field $E$ is the sum of $v \times B$ with the electrostatic field $E_{s}$ which is produced to balance the polarization produced by the current due to $v \times B$, namely, to satisfy $\operatorname{div} \Sigma\left(E_{s}+v \times B\right)=0$ where $\Sigma$ is the electric conductivity tensor integrated over the thickness of the $\mathrm{E}$ region.

This electric field $E=E_{s}+v \times B$ causes the charged particles to drift in the direction of $E \times B$ (Hirono, 1950). In the $F$ region where the collision frequency is lower than the gyrofrequency for both ions and electrons, the drift motion is shared by ions and electrons. Since the electric field $E$ of $S q$ is directed eastward in low latitudes, the drift is directed upward and transports ions and electrons away from the peak of the F2 layer. This acts to reduce the electron density at the peak of the F2 layer. The effect is most pronounced in the geomagnetic equatorial region where the magnetic field $B$ is in the horizontal direction. Hence a minimum is expected to appear at the geomagnetic equator in the latitudinal distribution of $f o \mathrm{~F} 2$. The equatorial anomaly of $f o \mathrm{~F} 2$ was explained in this way (Hirono and Maeda, 1954). 


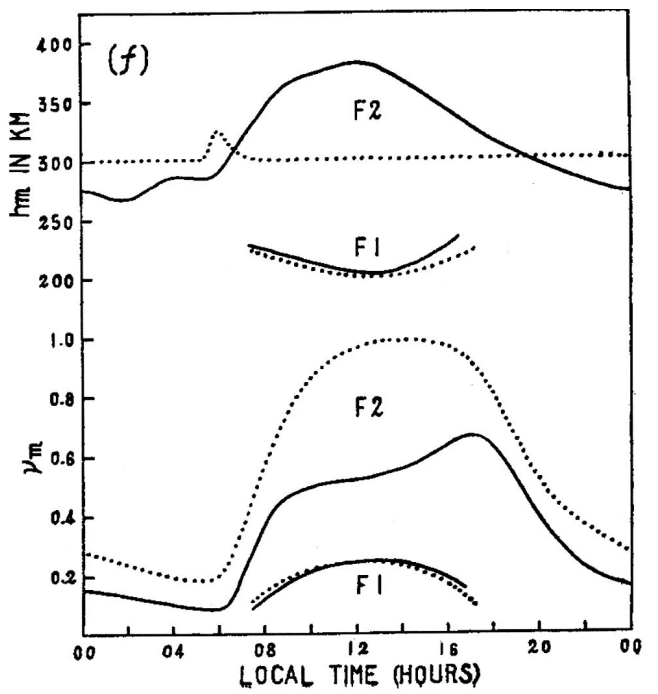

Figure 2. Theoretical models of the daily variation of the F2 layer at the geomagnetic equator. In the model where the vertical drift is taken into account (solid curve) the depression in density that had been observed as "equatorial anomaly" was reproduced, while the Chapman layer (dashed curve) was peaked at noon. Top: virtual height and bottom: electron density at the F2 peak (Hirono and Maeda, 1954).

This was demonstrated in Fig. 2 where daily variations of height (top) and electron density (bottom) at the peak of the equatorial F2 layer were compared between the above model (solid lines) and the Chapman model (dashed lines). The above model gave higher height and lower density at the F2 peak in the daytime than the Chapman model in agreement with observations. (Horizontal diffusion was not incorporated in their model.)

\subsection{Equatorial enhancement of magnetic variations}

In the $\mathrm{E}$ region where the drift motion of ions is impeded by collisions and only electrons can drift, the $E \times B$ drift produces a downward current. In other words, the Hall current flows downward under the eastward electric field and the northward magnetic field. The downward current builds up negative charges at the top of the $\mathrm{E}$ region and the polarization produces an upward electric field. The Hall current due to the upward electric field adds to the eastward Pedersen current generated by the eastward electric field that is originally imposed, so that the eastward current $J_{\text {East }}$ is given by $J_{\text {East }}=\left(\Sigma_{\mathrm{P}}+\Sigma_{\mathrm{H}}^{2} / \Sigma_{\mathrm{P}}\right) E_{\text {East }}$, where $\Sigma_{\mathrm{P}}$ and $\Sigma_{\mathrm{H}}$ represent Pedersen and Hall conductivities, respectively. Figure 3 shows the ratio between $\left(\Sigma_{\mathrm{P}}+\Sigma_{\mathrm{H}}^{2} / \Sigma_{\mathrm{P}}\right)$ and $\Sigma_{\mathrm{P}}$ against latitude. Case $\mathrm{A}$ is for the case where electrons carry negative charges while case $\mathrm{C}$ is for a hypothetical case where negative ions do so. For the relevant case A, a pronounced peak is seen at the equator. The equatorial enhancement was attributed to this effect (Hirono, 1952).

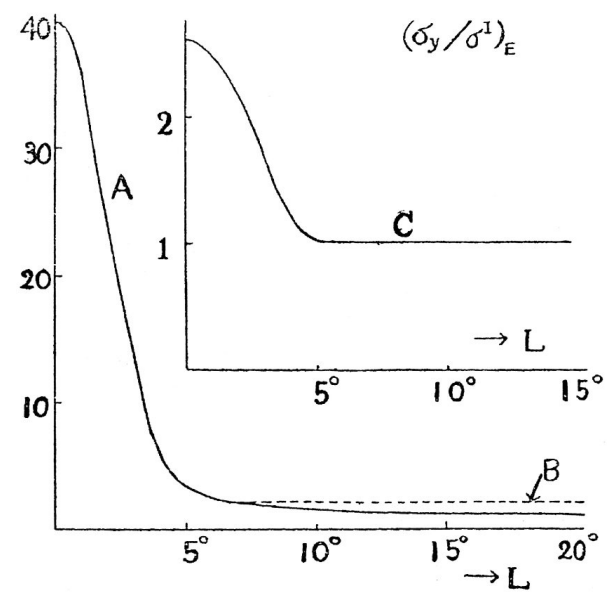

Figure 3. Enhancement of the electric conductivity along the equator when the vertical polarization field produced by Hall current is taken into account. The model $\mathrm{A}$ is the relevant case; $\mathrm{C}$ is a reference model where carriers of the negative charge are assumed to be negative ion (Hirono, 1950).

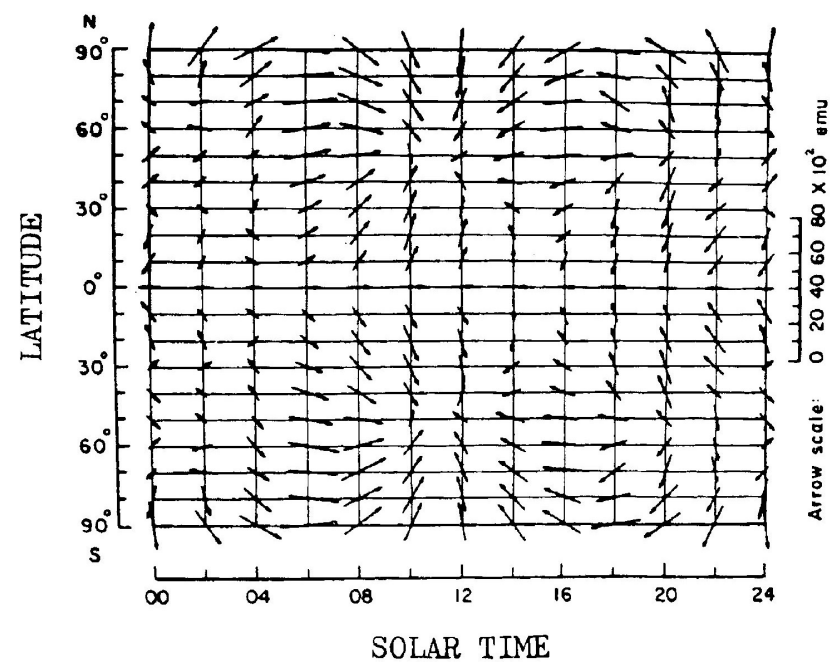

Figure 4. Tidal wind system in the E region derived from the geomagnetic $S q$ variation (Maeda, 1955).

\subsection{Tidal wind system in the E region}

On the basis of the dynamo theory, the wind system in the ionosphere was derived from the observation of the $S q$ current system. Using the current density $J$ obtained from $S q$ and the conductivity model, $J=\Sigma\left(E_{s}+v \times B\right)$ was solved for $E_{s}$ and $v$ using the conditions $\operatorname{div} J=0$ and $\operatorname{rot} E=0$. In an earlier calculation $v$ was assumed to be derivable from a potential (Maeda, 1955) but a more general expression for $v$ including the rotational component was considered later (Kato, 1957). Figure 4 shows the distribution of the tidal wind velocity obtained by the former author. In both cases, the tidal motion at the ionospheric height was found to be 
Average Storm-time Variation of $K_{p}$ and $D\left(F_{2}\right)$
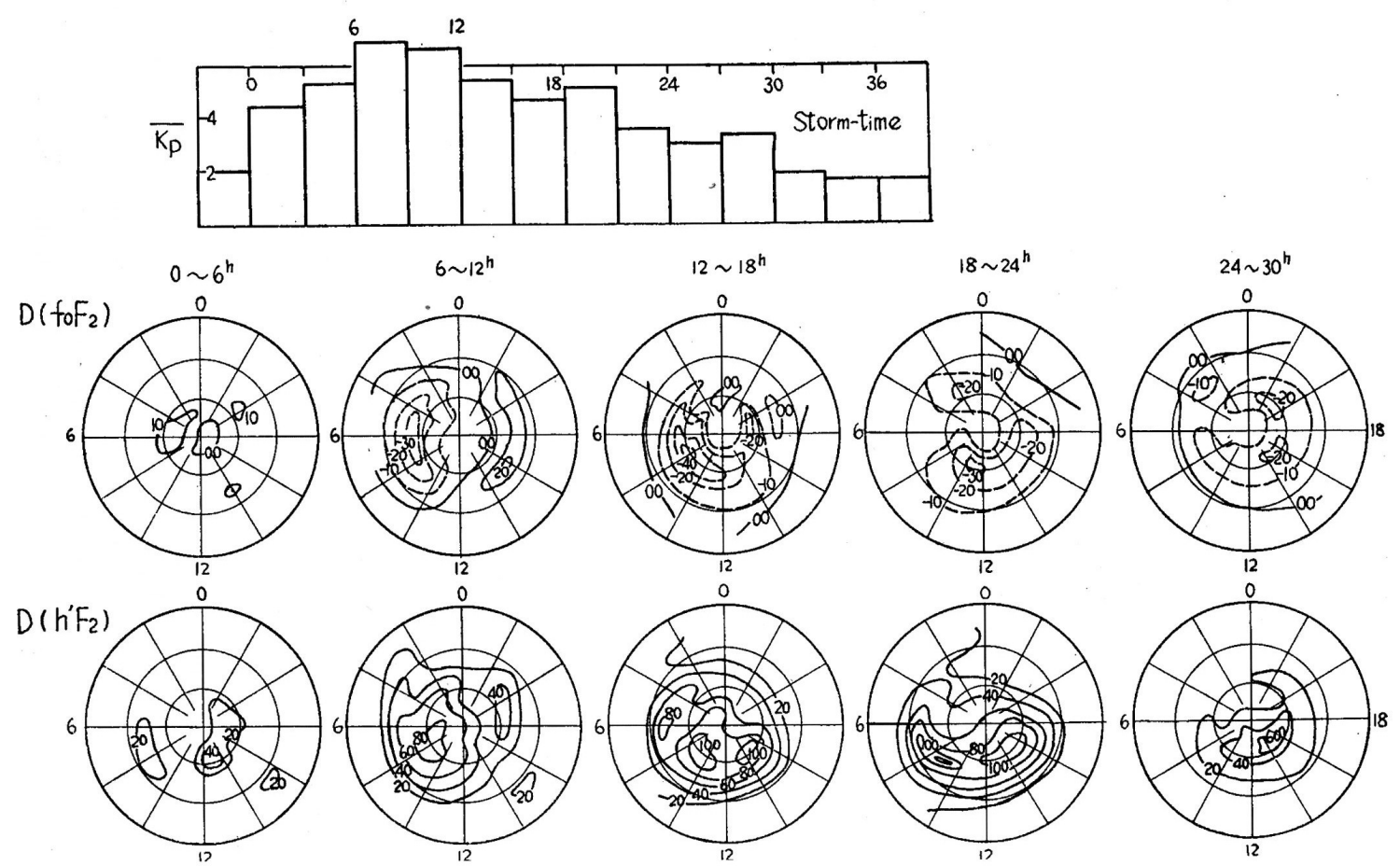

Figure 5. Ionospheric disturbance in the F2 layer obtained by averaging ten storms in 1950-1951 every six hours after the beginning. Top: Kp index, middle: percentage deviations of $f_{o} \mathrm{~F} 2$, and bottom: deviations of $h^{\prime} \mathrm{F} 2$ in km (Obayashi, 1954).

predominantly diurnal. This made a marked contrast to the atmospheric tidal oscillation observed on the ground where the semi-diurnal component was dominant. The difference in predominant modes of the tidal oscillation between ground and ionospheric heights posed a serious question.

\section{Disturbances}

\subsection{Ionospheric disturbance in the F2 layer}

Disturbances in the F2 layer were studied extensively (Uyeda, 1949). Worldwide morphology of the disturbances was constructed by superposing the observations of ten typical ionospheric storms during 1950-1951 (Fig. 5). The disturbance in $f o \mathrm{~F} 2$ is characterized by bifurcation between positive and negative changes. The positive and the negative regions appear on the evening-night and the morning-day sides, respectively, and then the negative region expands, spreading the decreases of $f o \mathrm{~F} 2$ to the global scale (Obayashi, 1954).

Attempts were made to interpret the disturbance observed in the F2 region in terms of the drift motion. The drift velocity was derived from the electric field obtained from the simultaneous measurement of the magnetic field. In Fig. 6, calculated values of $h^{\prime} \mathrm{F} 2$ and $f o \mathrm{~F} 2$ (B) are compared with the observed deviations from quiet times (A) at Washington D.C. for two intervals of $48 \mathrm{~h}$. Dashed curves that represent $\Delta f o \mathrm{~F} 2$ seem to show fair agreement between (A) and (B) in the cases of negative changes in $f o F 2$. Solid curves representing $\Delta h^{\prime} \mathrm{F} 2$ seem to differ but when the effect of retardation of the sounding radio wave is taken into account (yielding solid curves with small circles) the agreement seems improved. However, the calculated and the observed values of $\Delta f o \mathrm{~F} 2$ do not agree in the cases of positive changes of $f o \mathrm{~F} 2$ (Sato, 1957). In the light of the findings in later years that F2 storms involve several mechanisms, it has to be considered reasonable that the drift alone was not able to fully explain the observations.

\subsection{Magnetic disturbances and the dynamo theory}

Geomagnetic bays and elementary disturbances that constitute magnetic storms were compared with the popular model where the electromotive force was the same dynamo field as in quiet times but the electric conductivity was locally enhanced along the auroral zone. But the model did not agree with the observation; there was a very significant difference in phase (that means the direction of the current system in the frame of local time) amounting to $110^{\circ}-150^{\circ}$ (Nagata and Fukushima, 1952; Fukushima and Oguti, 1953).

Viewed from the vintage point of the present, it is amazing how broadly and deeply the success of the dynamo theory in interpreting the quiet-time features of the ionosphere 


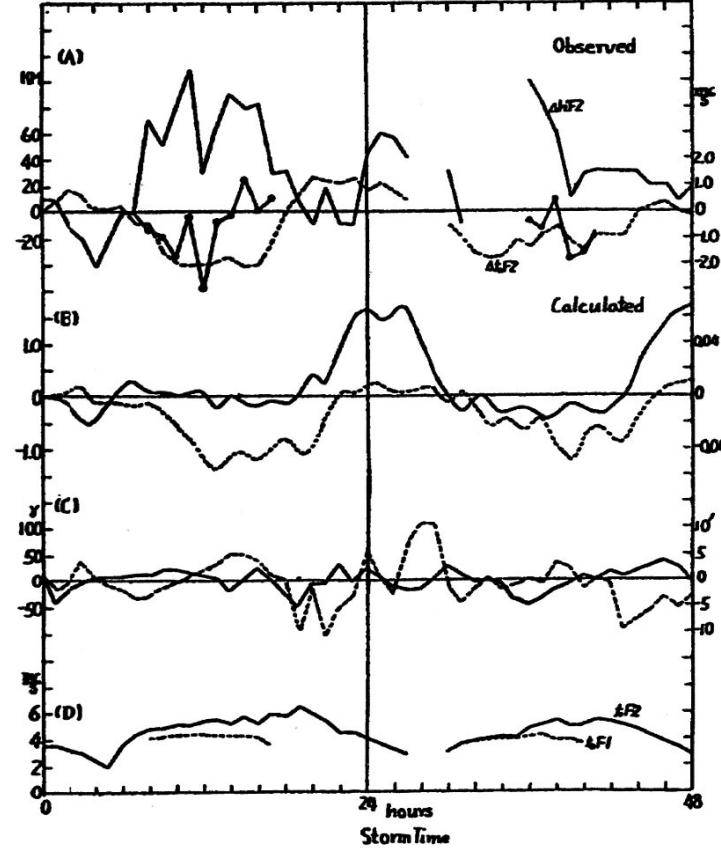

(a) Washington, $0^{h}$, Sep. 7, 1952

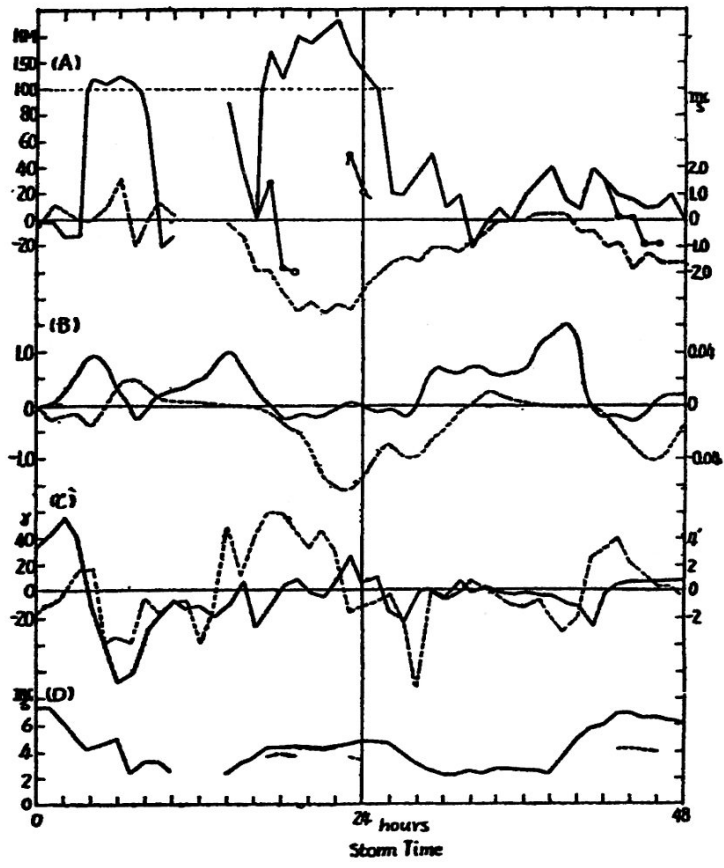

(b) Washington, 16 , Feb. 23, 1952

Figure 6. Comparison between (A) the observed and (B) the calculated variations of the F2 layer at Washington during ionospheric disturbances. The bottom panels show observations of (C) the magnetic field and (D) the ionosphere (Sato, 1957).

influenced the research in these days. It was even suggested that winds in the $\mathrm{D} /$ lower $\mathrm{E}$ layer and in the upper $\mathrm{E}$ layers have different configurations so that the transition of the current system from $S q$ to SD is caused by the switch of the current layer from one to the other (Matsushita, 1949; Nagata et al., 1950). Combination of the wind-driven dynamo and a localized enhancement in the ionospheric conductivity was invoked also to explain the preliminary impulse of $\mathrm{SC}^{*}$ (Nagata and Abe, 1955).

The enhancement in the electric conductivity in high latitudes was attributed to the ionizing effect of the impinging solar corpuscles. The "corpuscular stream" in these days corresponded broadly to what we call the solar wind today, but the properties attributed to them were not entirely the same. (1) It was supposed that the corpuscular stream was emitted from the sun for some limited intervals or from some limited locations. (2) As a typical speed, values of $1000-1500 \mathrm{~km} / \mathrm{s}$ were often quoted. This was partly because the sudden commencement of magnetic storms was attributed to the encounter with the corpuscular stream itself rather than to the impact of the shock wave that propagated ahead. (3) Some of the corpuscles were supposed to penetrate deep into the geomagnetic field. This accessibility should have been taken as a very serious problem since a strong energy dependence was already established by Stormer's theory of the motion of charged particles in the geomagnetic field. (4) Dynamical effect of the momentum of the corpuscular flow was not considered.

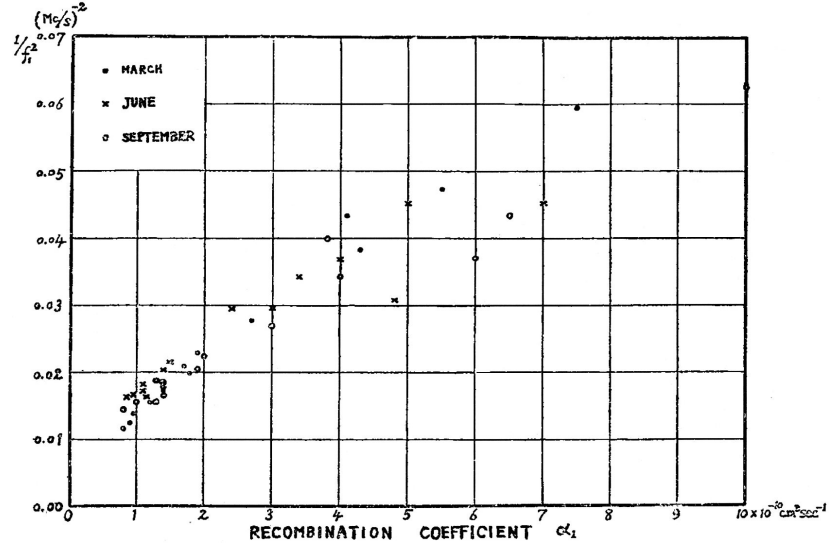

Figure 7. Horizontal axis is the coefficient that is obtained by fitting the recombination model and vertical axis is the inverse of $(f o \mathrm{~F} 2)^{2}$. There should not be a linear relation if the recombination model were applicable (Yonezawa, 1951).

\section{Formation of the F2 layer}

According to the classical theory of formation of the ionospheric F2 layer by Chapman, electrons are supposed to be lost by recombination. This means that the rate of the loss is proportional to the square of the electron density. However, when the apparent recombination coefficient was derived from observations at sunset, this was found to depend on the 


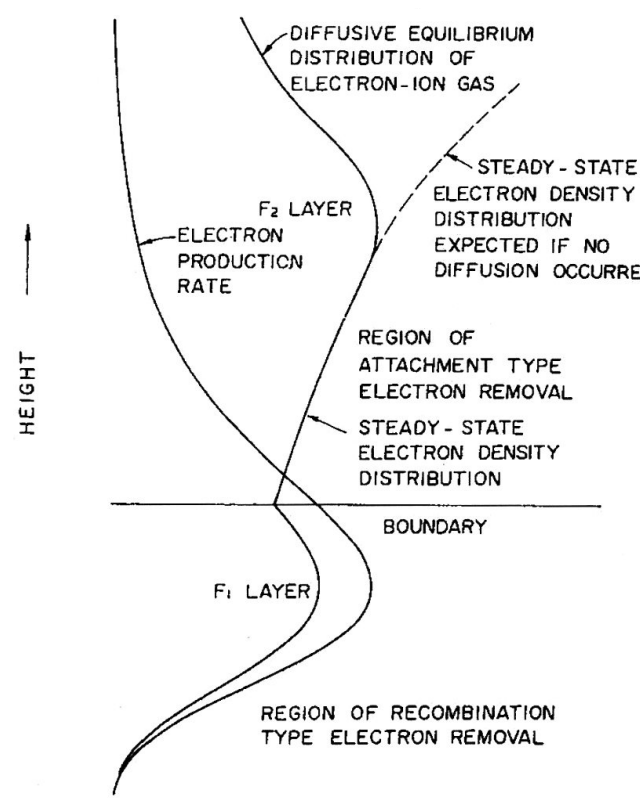

(a) BOUNOARY IS ABOVE THE PEAK OF ELECTRON PRODUCTION RATE

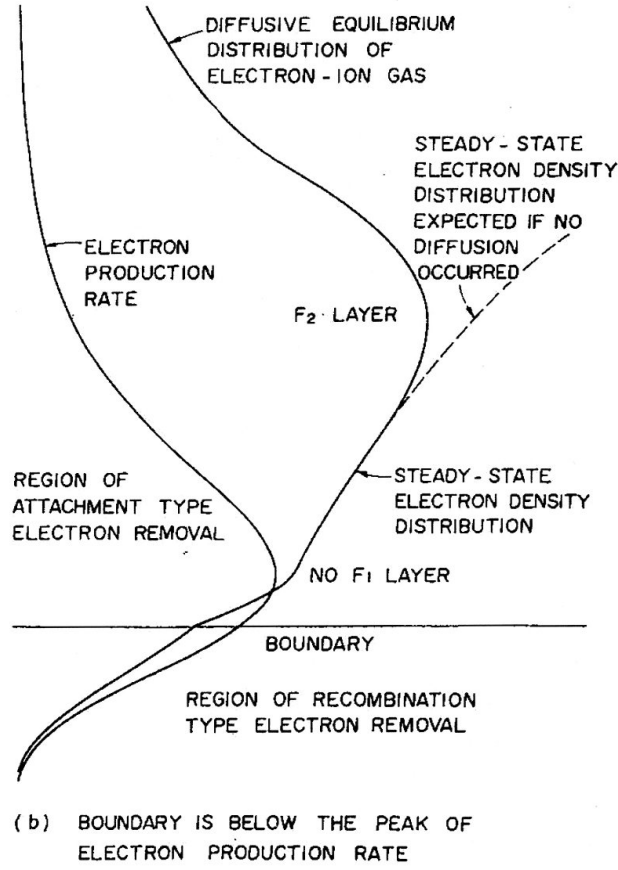

Figure 8. Schematic illustrations of the height distribution of the electron density. See text. (With kind permission from Springer Science + Business Media: Space Sci. Rev., Theory of formation of the ionosphere, 5, 1966, 3-56, Yonezawa, T., Fig. 5.)

maximum electron density $N_{\max }$ of the F2 layer (Fig. 7). The proportionality of the presumed recombination coefficient to $1 / N_{\max }$ suggested that the electrons are lost by attachment, rather than by recombination (Yonezawa, 1951).

Based on this finding, a new theory of the F2 layer formation was developed. It was argued that the electron loss proceeds in two steps. The first is the charge transfer between atomic ions of oxygen and neutral molecules of oxygen or excited nitrogen molecules. The second step is the dissociative recombination between the resulting molecular ions and electrons (Yonezawa, 1955). At higher altitudes in the $\mathrm{F}$ region the first step, that is, attachment, is the ratedetermining, while at lower altitudes the second step is the rate-determining because of rapid decreases in the number densities of $\mathrm{N}_{2}$ and $\mathrm{O}_{2}$ with height.

This reaction alone cannot produce the density peak of the F2 layer. The loss rate of electrons by attachment is proportional to the density of molecules $\left(\mathrm{N}_{2}\right.$ and $\left.\mathrm{O}_{2}\right)$ while their production rate is proportional to the density of atoms $(\mathrm{O}$ or $\mathrm{N}$ ), and the electron density is proportional to the ratio of the latter to the former. Since density of atoms decreases more gradually with height as compared to molecules, the above ratio increases monotonically with height and no peak would result. The decrease of the attachment coefficient with height enhances this tendency.

This can be seen in Fig. 8 which schematically illustrates the distribution of the electron density with height. Two panels correspond to different cases of the transition height of the loss mechanism from recombination to attachment; it oc- curs at a relatively high altitude in the left-hand case and at a lower altitude in the right-hand case. In both cases, the dashed curves that extend to high altitudes represent the density distribution is expected when the loss is by attachment.

However, Yonezawa (1956) proposed that the peak of the electron density at F2 could still be produced by the effect of diffusion in the earth's gravitational field. Electron-ion pairs generated in the upper portion of the F2 layer come down quickly by diffusion, so that the distribution takes a form of the diffusive equilibrium. When this effect is incorporated, the electron distributions have a peak as drawn by solid curves in Fig. 8. The model is compared with observations in Fig. 9 where variations of the F2 maximum electron density with latitude are compared between the model (solid curve) and the observations (dots). They agree very well except in the equatorial region where the diffusion in the horizontal direction need be incorporated. Thus, a new theory of the formation of the F2 layer was established (Yonezawa, 1956).

\section{Plasma waves}

\subsection{Ray paths of VLF waves}

While the ionospheric sounding used high frequency radio waves in the HF range, radio emissions of natural origin were observed in the VLF range. In this frequency range the whistler atmospherics are produced by lightening discharges. Extraordinary ray component of these waves penetrate the 


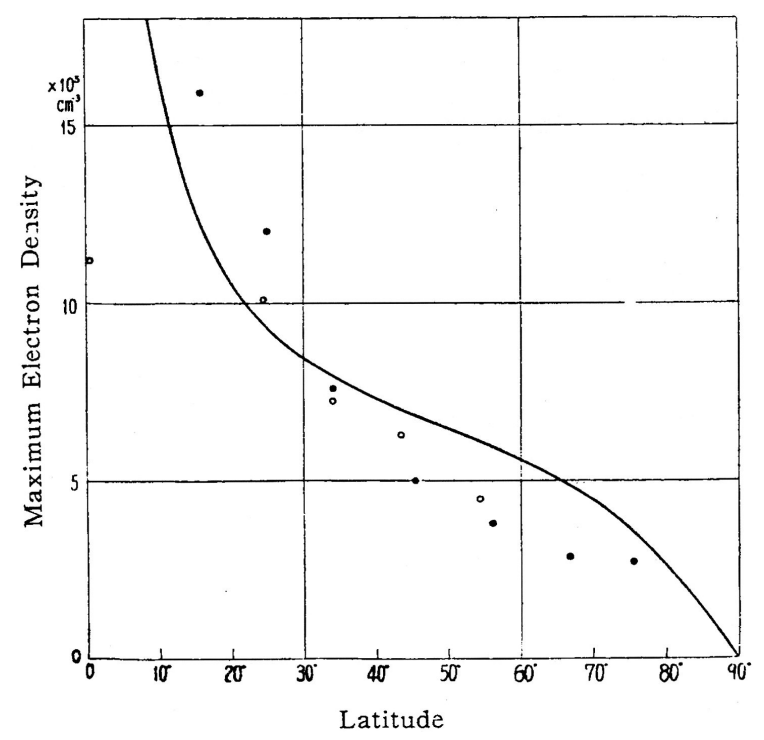

Figure 9. Comparison between the observed and the calculated variations of the electron density at the peak of F2 layer with latitudes (Yonezawa, 1956).

ionosphere and propagate through the outer region along geomagnetic field lines. In a classical paper published in 1953 Storey found that the plasma extended above the F2 layer and derived the electron density of $400 \mathrm{~cm}^{-3}$ at a height of two earth-radii by analyzing the frequency dispersion of the whistler atmospherics.

His work demonstrated that VLF waves of natural origin could be a powerful tool for investigating the physics of the outer ionosphere. (The term magnetosphere not yet existed at the time.) In order to provide the basis for interpreting the whistler observations Maeda and Kimura (1956) performed an exact calculation of ray paths of whistlers. Rays were emitted from several locations in the ionosphere; the highest $\mathrm{L}$ value of the emitting site was 4.5. Tracing was performed graphically using Fermat's principle.

The result of their ray tracing is shown in Fig. 10. They found that for the sources that are located at relatively low geomagnetic latitudes the rays arrive at a latitude higher than the source latitude. For the sources located at high latitudes, on the other hand, the rays are more symmetric but deviate appreciably from geomagnetic field lines. This paper has played a pioneering role to later studies of VLF waves as it became known that there are many other kinds of wave sources that are generated by wave-particle interactions in the magnetosphere.

\subsection{Resonance model of magnetic pulsations}

Waves in the ULF range seen in magnetograms are called magnetic pulsations. They are classified into several types according to frequency, regularity and spatial distribution. Giant pulsations (which are called Pc5 according to the cur-

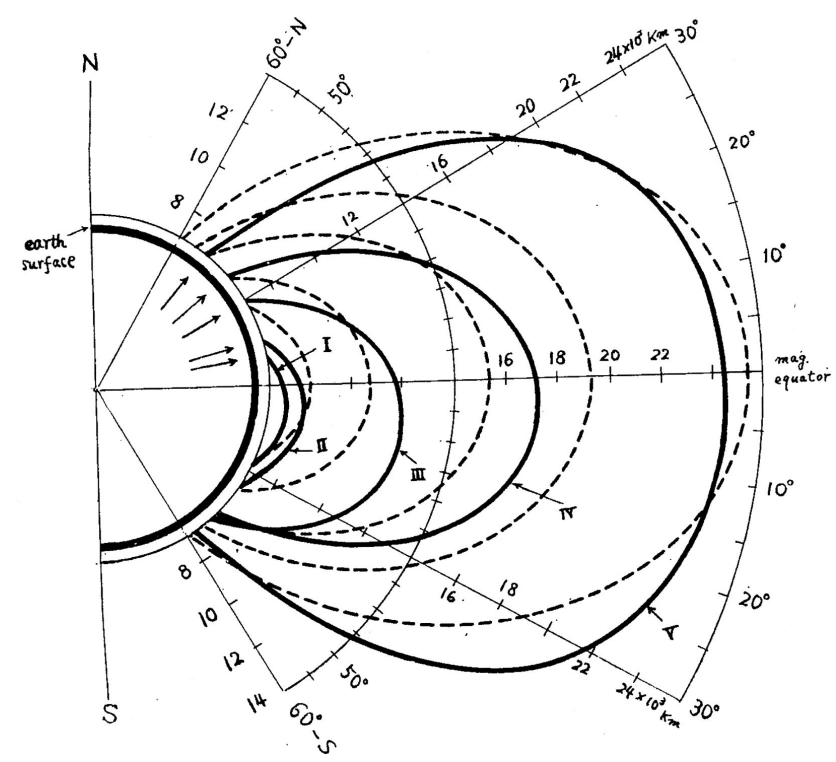

Figure 10. Calculated ray paths of VLF waves (solid curves) and geomagnetic field lines (dashed curves) (Maeda and Kimura, 1956).

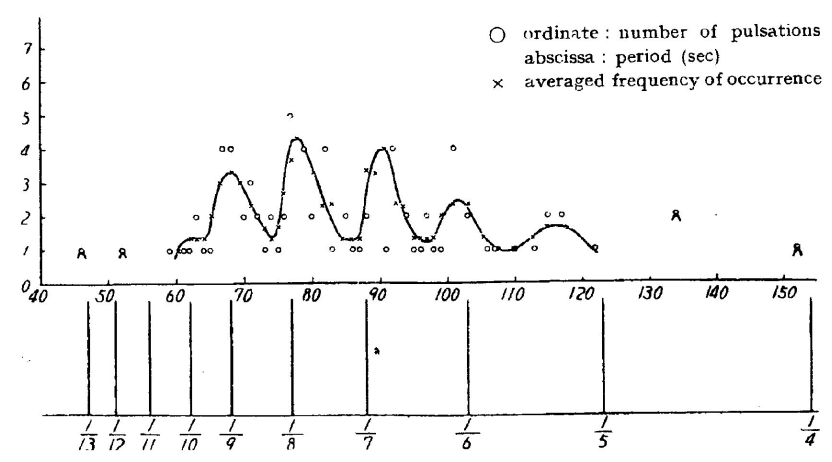

Figure 11. Harmonic structure of the spectrum of giant pulsations (Kato and Watanabe, 1954). Top: observed spectrum, and bottom: comparison with harmonics.

rent nomenclature) are characterized by amplitudes of $15-$ $30 \mathrm{nT}$, periods of several minutes, and occurrence in auroral latitudes. Kato and Watanabe (1954) found that the spectra of these pulsations had harmonics at $1 / n$ of the fundamental period (Fig. 11), and suggested that they were resonant oscillations of magnetohydrodynamic waves excited by external disturbances. This suggestion was published in the same year as Dungey's well known report from the Pennsylvania State University. It was unfortunate that Kato and Watanabe do not seem to have been aware of Storey's paper on the plasma density above the ionosphere. Later Obayashi and Jacobs (1958) calculated the resonance period of pulsations by using a realistic model of the density distribution and found that the latitude dependence of the period can be well explained by this model. 

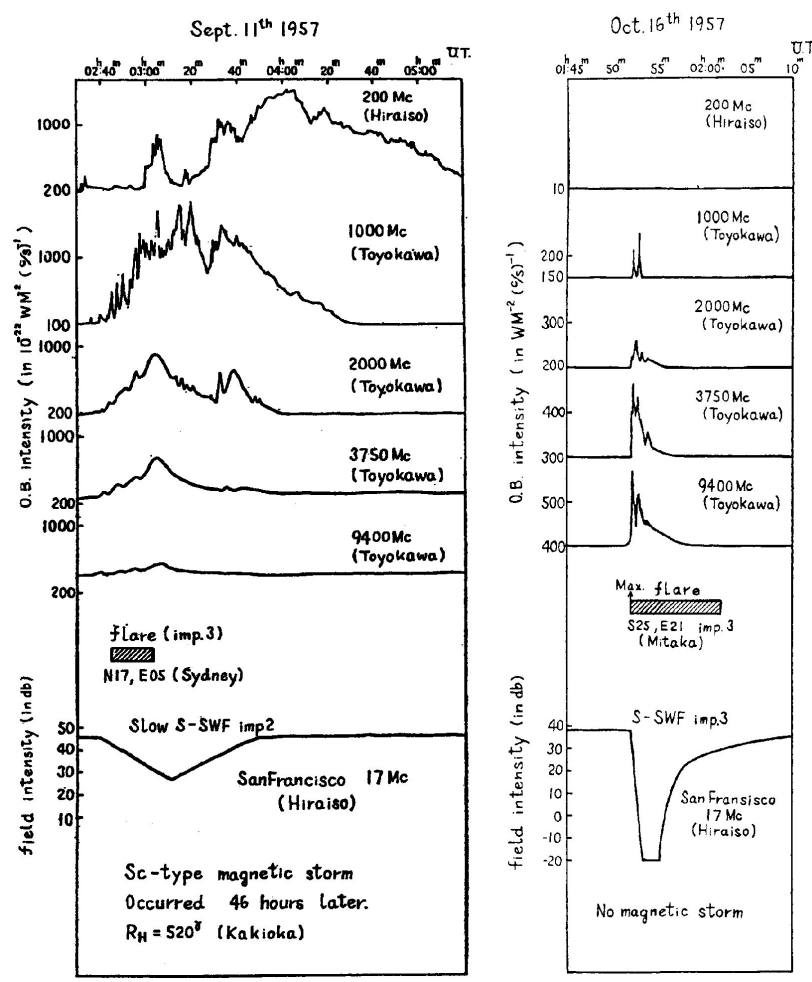

Figure 12. Comparison of spectrum of solar radio bursts (five top curves), interval of flare (shaded box), field intensity of intercontinental radio transmission indicating the strength of SWF (bottom curve) and property of magnetic disturbance (handwritten at the bottom). Left: a case of low frequency burst and magnetic storm, and right: a case of high frequency burst and no magnetic storm (Sinno and Hakura, 1958).

\section{Solar terrestrial relation}

From the intensive efforts during the IGY to forecast geomagnetic and ionospheric storms, two important phenomena were identified that preceded the storms. One was the typeIV solar radio outburst and the other was the blackout in the ionospheric radio propagation across the polar cap. When solar radio outbursts from $200 \mathrm{MHz}$ to $9400 \mathrm{MHz}$ were compared with the ionospheric disturbances, it was found that the spectral property of the outbursts was related to the character of the disturbance they produced. As shown in Fig. 12, outbursts whose spectral intensity increased toward lower frequencies (left panel) tended to cause storms but not SWFs. (Short Wave Fadeout (SWF) means sudden increase in the absorption in the D and E regions due to enhancement in the ionization.) In contrast, the outbursts whose intensity increased toward higher frequencies (right panel) almost always caused SWFs but no storms. There were also cases where two types were combined (Sinno and Hakura, 1958). Outbursts with the low frequency component were identified with the synchrotron radiation emitted by energetic electrons and were called Type-IV. The source location of Type-IV ra- dio outburst moved outward through the corona. Hence the occurrence of solar eruptions that eject corpuscular clouds, that is, "coronal mass ejections" according to the current nomenclature, could be monitored from the ground by looking out for this type of the outburst.

The polar cap blackout is caused by the abnormal enhancement in the radio wave absorption in the polar cap. It was found that the polar cap blackouts began several hours after the solar flares accompanied by major Type-IV radio bursts. An example is shown in Fig. 13. The absorption was caused by precipitation of energetic particles having energies of the order of 10-100 MeV which were high enough for entering the geomagnetic field and producing anomalous ionization in the D region. Since it began well ahead of a storm, the polar cap blackout served as a very useful tool for forecasting disturbances in the radio propagation. At the onset of magnetic storms the region of the blackout spread to auroral latitudes and took a spiral-like shape centered on the morning side (Hakura, Takenoshita, and Otsuki, 1958).

The delay time between the Type-IV radio outburst and the beginning of the polar cap blackout, that is, the propagation time of energetic particles having energies of $10-100 \mathrm{MeV}$, depended on the solar longitude of the outburst site and was shorter for outbursts that occurred on the western side of the solar central meridian than those on the eastern side: see Fig. 14. This was interpreted in terms of the spiral structure of the interplanetary magnetic field where the field lines that reach the Earth originate from the western side of the central meridian. Particles from the west could stream directly along the field lines while those from the east had to diffuse across field lines. No such asymmetry was found for the delay between the Type-IV outburst and the onset of storms (Obayashi and Hakura, 1960b).

The above sequence of events, namely, (1) solar flare accompanied by Type-IV burst, (2) polar cap absorption, and (3) onset of magnetic and ionospheric storm at the arrival of the solar corpuscles, was demonstrated by numerous case studies, and it presented a powerful tool for warning the disturbance in the radio propagation. This study may have been symbolic of a happy marriage between the curiosity-driven basic research and the applied research to improve the social infrastructure.

It was also recognized that there were exceptions to the sequence of events (1), (2) and (3). There were events where (1) type-IV burst was followed by (2) polar cap absorption, but magnetic storm did not follow and absorption did not spread to auroral latitudes. The only signature of the magnetic disturbance was a sudden impulse (Obayashi and Hakura, 1960a). There were also events where magnetic disturbance was produced several hours before a sudden impulse (Hakura et al., 1961). These suggested that some of the key factors in the solar terrestrial relation remained unidentified at the time. 


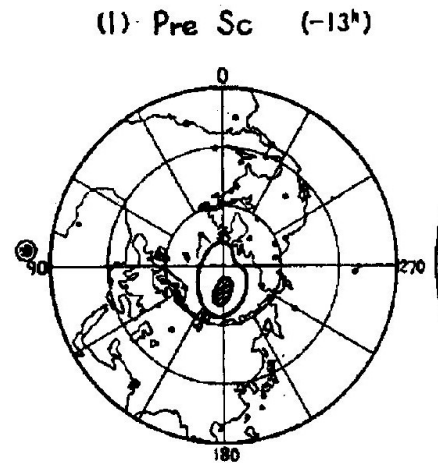

(5) Initial Phase (+2n)

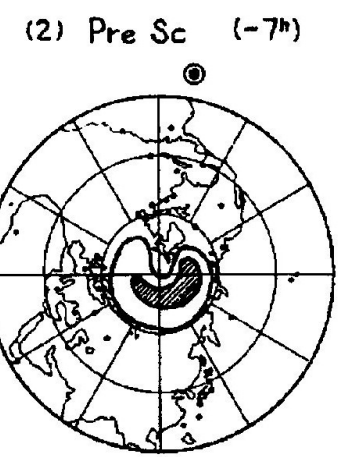

(6) Main Phase (+6n)
(3) Pre Sc $\left(-45^{m}\right)$

(4) Sc
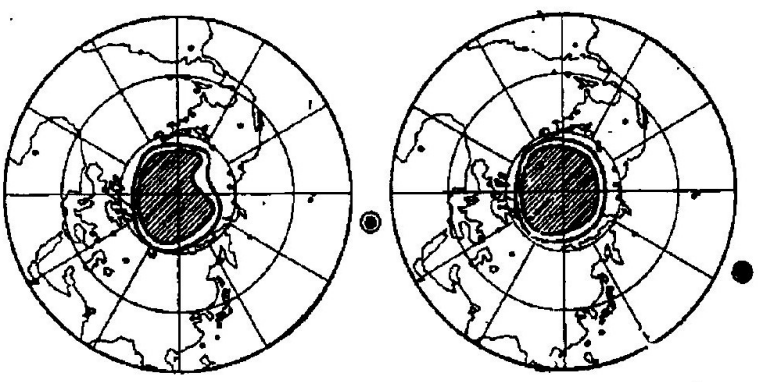

(7) Main Phase $\left(+9^{h}\right)$

18) Last Phase (+11")
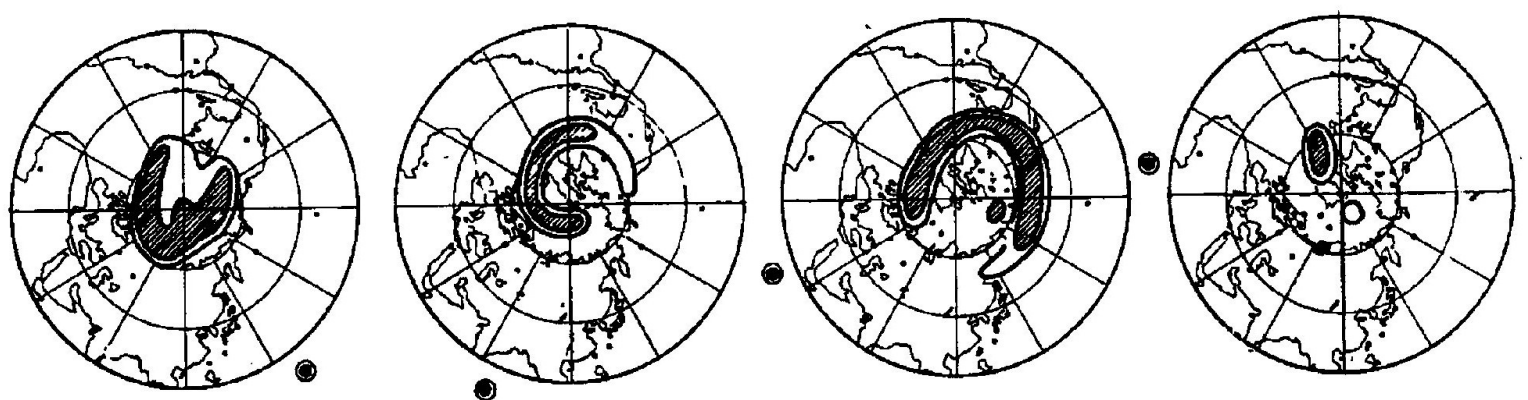

Figure 13. Sequence of the ionospheric absorption following a solar radio burst. Times in the parenthesis are counted from the geomagnetic storm sudden commencement. (2) Absorption begins several hours before the storm onset, (3) fills the polar cap, and (5 to 8 ) moves to the auroral zone after the onset (Obayashi and Hakura, 1960a).

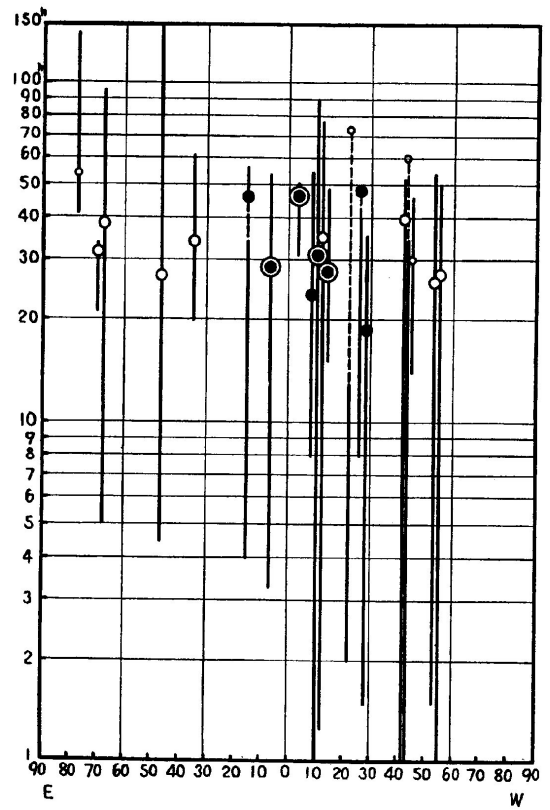

Figure 14. Heliographic distribution of time delays to the polar cap absorptions (where solid lines are drawn throughout their durations) and of geomagnetic storms (where dots indicate the storm intensity) from the Type-IV radio outburst (Obayashi and Hakura, 1960b).
The auroral zone absorption was also attributed to the direct entry of solar particles and the shift to lower latitudes was interpreted to be due to decrease in the magnetic field by the ring current. This interpretation had to be changed when more became known about the internal dynamics of the magnetosphere and the auroral zone absorption was found to result from the precipitation from inside the magnetosphere.

\section{IGY programs of sounding rocket and Antarctic research}

The International Geophysical Year (IGY, 1957-1958) gave a great impetus to the geophysical research worldwide. Japan was no exception. Most significantly, programs of the sounding rockets and the Antarctic research were initiated on this occasion.

The rocket program started in 1955 with the development of the rockets themselves. The first observation of the ionosphere was made in September 1960 with a Langmuir probe. Its record (Fig. 15) shows the electron density profile at middle latitude up to the height of $\sim 200 \mathrm{~km}$ in the afternoon. Maximum electron densities of $\mathrm{E}$, Es and $\mathrm{F}$ layers obtained by the vertical incidence sounder at a nearby ground station are also shown for reference. It is not difficult to imagine the excitement at seeing for the first time the topside structure that had been hidden to traditional sounders. 


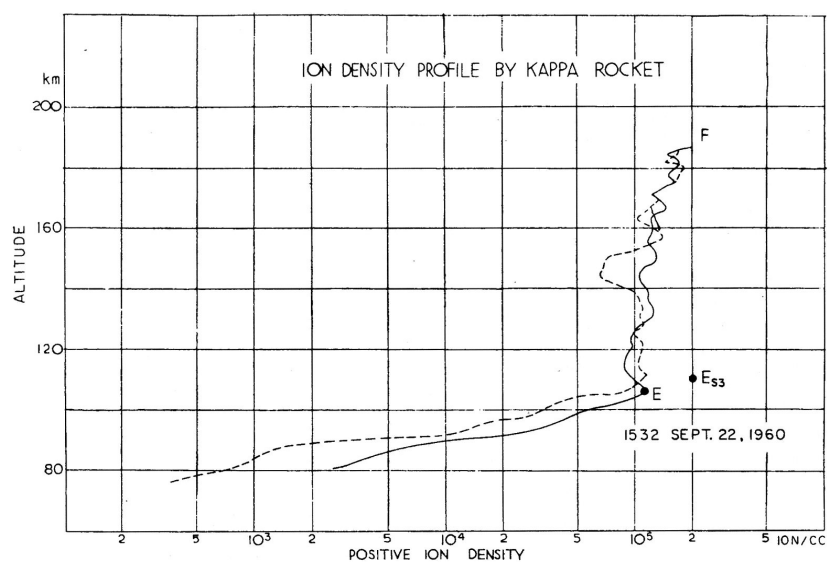

Figure 15. Electron density profile obtained at 15:32 JST, 22 September 1960 by a K-8 rocket launched from Michikawa, Japan. (Reprinted from Planet. Space Sci., 9, Maeda, K. and Hirao, K.: A review of upper atmosphere rocket research in Japan, 355-369, Copyright 1962, with permission from Elsevier.)

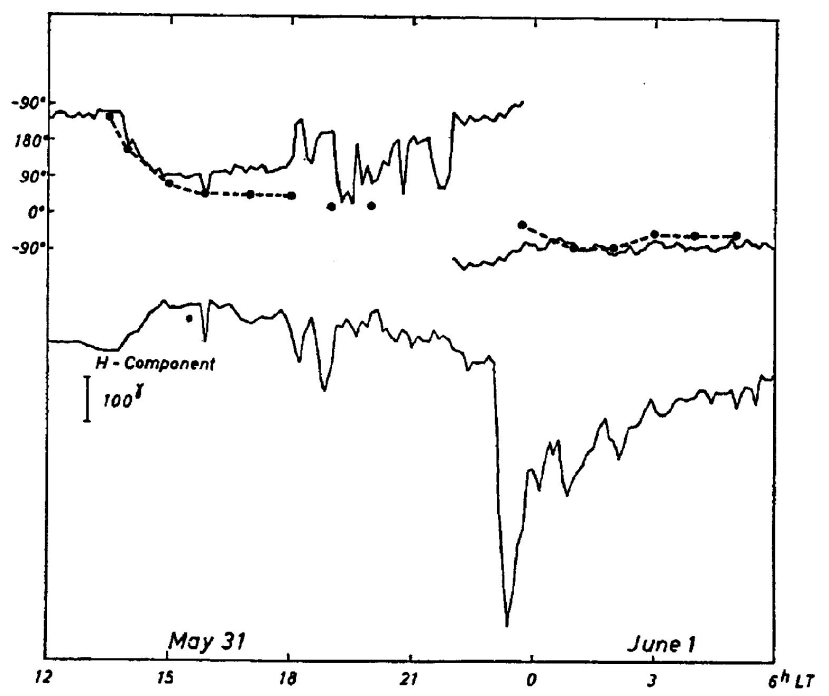

Figure 16. Comparison between directions of ionospheric current and lower boundary of aurora (Oguti, 1960).

In the Antarctic, the Japanese Syowa Base was established in January 1957. Observations of magnetic field, ionosphere, aurora and airglow were basic elements of the program. Since Syowa is located under the auroral zone it represented an ideal platform for studying aurora and its related phenomena. A full set of instruments were deployed during the wintering in 1959. An example of the observed relation between aurora and geomagnetic disturbance is shown in Fig. 16. The solid line in the upper part of the figure is the direction of the overhead current flow and the dots show the direction of the lower boundary of aurora. Their excellent agreement demonstrated that the ionospheric current was flowing along the latter (Oguti, 1960).

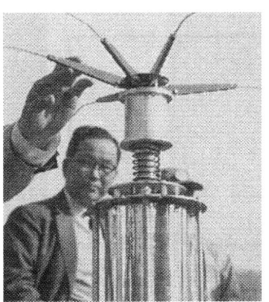

Ken-ichi Maeda

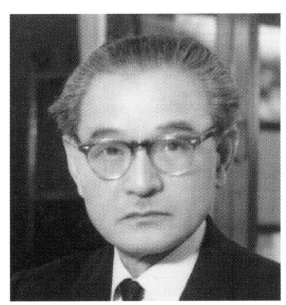

Mankichi Hasegawa

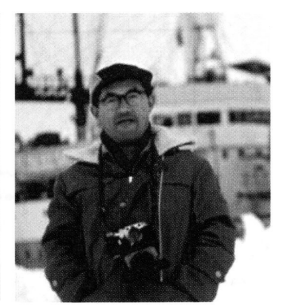

Takesi Nagata
Figure 17. Japanese leaders at the time of IGY: K. Maeda (19091995), M. Hasegawa (1894-1970) and T. Nagata (1913-1991). All the pictures were dated between 1955 and 1958.

The Japanese community in geomagnetism and ionospheric physics has benefitted from having enthusiastic and competent leaders. Figure 17 presents pictures of three prominent leaders at the time of the IGY. Ken-ichi Maeda (left), an ionospheric physicist, promoted experiments in space by organizing developments of rocket-borne scientific instruments. Takesi Nagata (right), a geomagnetician, led the Antarctic Research Expedition and established the Syowa Base. Mankichi Hasegawa (center), a geomagnetician who had founded the Japan Society of Geomagnetism and Geoelectrity, chaired the Japanese IGY Committee. We have a wealth of research programs that have been developed upon the heritage built by them. The scientific satellite programs at ISAS/JAXA, Antarctic Research conducted by the National Institute of Polar Research and the atmospheric radar program at the Institute of Sustainable Humanosphere at Kyoto University are the outstanding examples. CAWSES is also along the line of international programs in which the Japanese community has been deeply involved.

\section{Epilogue}

In the preceding Chapters I have noted that three major questions were raised from the space weather or solar terrestrial research before 1960. The first is why the tidal wind in the ionospheric $\mathrm{E}$ region is predominantly diurnal while the semi-diurnal tide prevails in the lower atmosphere. The second is how the electric field can be generated in the magnetosphere and ionosphere by mechanisms other than the atmospheric dynamo. The third is what other factors govern the solar wind-magnetosphere interaction than the properties of the solar corpuscles themselves.

The diurnal mode of the tidal oscillation which is essentially confined in the ionosphere was found in the solution of the tidal equation when the eigen value (representing the thickness of the layer) is allowed to be negative. Such a mode does not propagate but decreases or increases exponentially in the vertical direction, that is, the oscillation is confined to a region where the energy is supplied. The solution of the first 
negative mode agreed very well with the pressure variation deduced from the geomagnetic $S q$ variation (Kato, 1966).

A new type of geomagnetic daily variations, $S_{q}^{p}$, was discovered by Kokubun (1964) in the sunlit polar cap on geomagnetically quiet days. The ionospheric current density corresponding to $S_{q}^{p}$ was too high to be generated by the combination of the dynamo electric field and the reasonable estimate of conductivity in the polar cap. Hence it was suggested that the electric field of $S_{q}^{p}$ was the counterpart of the magnetospheric convection that is generated by the force exerted from the solar wind as proposed by Axford and Hines; see also Nagata and Kokubun (1962).

The geomagnetic field was found to respond globally (that is, from the equator to the pole) to the north-south component of the interplanetary magnetic field (IMF). The magnetic variation which showed this feature seemed to be the same as $S_{q}^{p}$ when smoothed. Its correlation with IMF was so distinct that the peaks of respective variables matched with a time delay of several minutes. It was considered that the magnetic variation of this type, named DP2, reflects the time variability of the magnetospheric convection, and the excellent correlation with IMF suggests that the convection is driven by magnetic reconnection between the geomagnetic and interplanetary magnetic field lines; convection is stronger when IMF is closer to being southward and so is the magnetic disturbances produced in the magnetosphere as expected by Dungey's theory (Nishida, 1968). Thus the additional factor which exerts dominant role in the solar wind-magnetosphere interaction was identified to be the interplanetary magnetic field.

Once it was recognized that the ionosphere is coupled with the magnetosphere, it became untenable to use a model where the current was practically confined in the ionosphere. The field-aligned current that transports energy between the two regions had to be explicitly incorporated. An inversion method was developed to derive the electric field and the three-dimensional current system from observations of the magnetic disturbance field on the ground coupled with a realistic model of the conductivity distribution (Kamide et al., 1981). The method has become a common tool for the magnetospheric research that employ ground magnetograms.

Acknowledgements. I thank Kiyoshi Igarashi for providing me with valuable records of the ionospheric research in the wartime and the immediate postwar period. I also thank Iwane Kimura and Hiroshi Maeda for sharing their personal recollections with me. "M. Hasegawa and Geomagnetism and Geoelectricity" a monograph in Japanese by Hiroshi Nagano and Yasuharu Sanoo was a very useful summary of the research conducted by M. Hasegawa and his group at the Kyoto University.

Edited by: T. V. Kuznetsova

Reviewed by: G. P. Gregori and two other anonymous referees

\section{References}

Fukushima, N. and Oguti, T.: Polar magnetic storms and geomagnetic bays, Appendix I. A theory of SD field, Rept. Ionosph. Res. Jpn., 7, 137-146, 1953.

Hakura, Y., Takenoshita, Y., and Otsuki, T.: Polar blackouts associated with severe geomagnetic storms on Sept. 13th, 1957 and Feb. 11th, 1958, Rept. Ionosph. Res. Jpn., 12, 459-468, 1958.

Hakura, Y., Nagai, M., and Sano, Y.: Development of ionospheric and geomagnetic storms caused by solar corpuscular emissions, II. Polar blackouts, storm Es, and geomagnetic storms, Rept. Ionosph. Space Res. Jpn., 15, 14-28, 1961.

Hasegawa, M.: On the type of the diurnal variations of the terrestrial magnetism on quiet days, Proc. Imp. Acad. Tokyo, 12, 88-90, 1936.

Hatakeyama, H.: On the bay-disturbance in the terrestrial magnetic field, Geophys. Monogr. Cent. Meteorol. Obs., Tokyo, 12, 1566, 1938.

Hirono, K.: On the influence of the Hall current to the electrical conductivity of the ionosphere, J. Geomag. Geoelectr., 2, 1-8, 1950.

Hirono, K.: A theory of diurnal magnetic variations in equatorial region and conductivity of the ionospheric E region, J. Geomag. Geoelectr., 4, 7-21, 1952.

Hirono, M. and Maeda, H.: Geomagnetic distortion of the F2 region on the magnetic equator, J. Geomag. Geoelectr., 6, 127-144, 1954.

Kamide, Y., Richmond, A. D., and Matsushita, S.: Estimation of ionospheric electric fields, ionospheric currents, and field aligned currents from ground magnetic records, J. Geophys. Res., 86, 801-813, 1981.

Kato, S.: Horizontal wind systems in the ionospheric E region deduced from the dynamo theory of geomagnetic $S q$ variation, Part IV, J. Geomag. Geoelectr., 9, 107-115, 1957.

Kato, S.: Diurnal atmospheric oscillation, 1. Eigenvalues and Hough functions, J. Geophys. Res., 71, 3201-3219, 1966.

Kato, Y. and Watanabe, T.: A possible explanation of the cause of giant pulsations, Sci. Rept. Tohoku Univ., Ser. 5, 6, 95-104, 1954.

Kokubun, S.: Polar magnetic variations and their conjugacy, Ph.D. Thesis, University of Tokyo, 1964.

Maeda, H.: Horizontal wind systems in the ionospheric E region deduced from the dynamo theory of geomagnetic $S q$ variation, Part I. Non-rotating Earth, J. Geomag. Geoelectr., 7, 121-132, 1955.

Maeda, K. and Kimura, I.: A theoretical investigation on the propagation path of the whistling atmospherics, Rept. Ionosph. Res. Jpn., 10, 105-123, 1956.

Maeda, K. and Hirao, K.: A review of upper atmosphere rocket research in Japan, Planet. Space Sci., 9, 355-369, 1962.

Matsushita, S.: Circulatory motion in the ionospheric atmosphere and their relation to the S field of the terrestrial magnetism. II, J. Geomag. Geoelectr., 1, 41-47, 1949.

Nagata, T. and Fukushima, N.: Constitution of polar magnetic storms, Rept. Ionosph. Res. Jpn., 6, 85-97, 1952.

Nagata, T. and Abe, S.: Notes on the distributions of SC* in high latitudes, Rept. Ionosph. Res. Jpn., 9, 39-44, 1955.

Nagata, T. and Kokubun, S.: An additional geomagnetic daily variation field (Sqp-field) in the polar region on geomagnetically quiet 
day, Rept. Ionosph. Space Res. Jpn., 16, 256-274, 1962.

Nagata, T., Fukushima, N., and Sugiura, M.: Electro-dynamical behavior of the ionosphere region viewed from geomagnetic variation, J. Geomag. Geoelectr., 2, 35-44, 1950.

Nishida, A.: Coherence of geomagnetic DP 2 fluctuations with interplanetary magnetic variations, J. Geophys. Res., 73, 55495559,1968

Obayashi, T.: On the world-wide disturbances of the ionosphere, Rept. Ionosph. Res. Jpn., 8, 135-170, 1954.

Obayashi, T. and Jacobs, J. A.: Geomagnetic pulsations and the Earth's outer atmosphere, Geophys. J., 1, 53-63, 1958

Obayashi, T. and Hakura, Y.: Enhanced ionization in the polar ionosphere caused by solar corpuscular emissions, Rept. Ionosph. Space Res. Jpn., 14, 1-38, 1960a.

Obayashi, T. and Hakura, Y.: Propagation of solar cosmic rays through the interplanetary magnetic field, Rept. Ionosph. Space Res. Jpn., 14, 427-434, 1960b.

Oguti, T.: On the Earth storms V, Inter relations among the upper atmosphere disturbance phenomena over the polar regions, Rept. Ionosph. Space Res. Jpn., 14, 291-299, 1960.

Rishbeth, H.: The equatorial F-layer: progress and puzzles, Ann. Geophys., 18, 730-739, 2000,

http://www.ann-geophys.net/18/730/2000/.
Sato, T.: Disturbances in the ionospheric F2 region associated with geomagnetic storms, II. Middle latitudes, J. Geomag. Geoelectr., 9, 1-22, 1957.

Sinno, K. and Hakura, Y.: On the relation of solar eruptions to geomagnetic and ionospheric disturbances, I. On the power spectrum of solar radio outbursts, Rept. Ionosph. Res. Jpn., 12, 285-295, 1958.

Uyeda, H.: World-wide distribution and time variation of ordinary component critical frequency of F2 layer, Rept. Ionosph. Res. Jpn., 3, 1949.

Yonezawa, T.: An analysis of electron density variations in the F2 layer after sunset, Rept. Ionosph. Res. Jpn., 5, 1-12, 1951.

Yonezawa, T.: A consideration of the mechanism of electron removal in the F2 layer of the ionosphere, II, Rept. Ionosph. Res. Jpn., 9, 17-37, 1955.

Yonezawa, T.: A new theory of formation of the F2 layer, J. Radio Res. Lab., 3, 1-16, 1956.

Yonezawa, T.: Theory of formation of the ionosphere, Space Sci. Rev., 5, 3-56, 1966. 